\title{
O estado da arte em Recuperação de modelos tridimensionais por conteúdo através de uma Revisão Sistemática
}

\author{
Leila Cristina Carneiro Bergamasco ${ }^{1}$, Fátima L. S. Nunes ${ }^{1}$ \\ ${ }^{1}$ Laboratório de Aplicações de Informática em Saúde (LApIS) \\ Escola de Artes, Ciências e Humanidades - Universidade de São Paulo (USP) \\ leila.cristina@usp.br, fatima.nunes@usp.br
}

\begin{abstract}
The advancement of technology enables the construction of more complex information systems. One approach that has been developed in this context is the use of three-dimensional environments. Due to the large volume of images databases the implementation of efficient methods to perform the search for these models is necessar. The content-based retrieval technique is one of the techniques to this work. It has several algorithms for characteristcs extraction and use similarity function to compare different objects. This article presents results of a Systematic Review on three-dimensional model retrieval in order to analyze techniques, extractors, similarity functions and databases used.
\end{abstract}

Resumo. O avanço da tecnologia possibilita a construção de Sistemas de Informação mais complexos. Uma das abordagens que tem sido desenvolvida nesse sentido é o uso de ambientes tridimensionais. Devido ao grande volume de dados nas bases de imagens é necessária a implementação de métodos eficientes para efetuar a busca desses modelos. Uma das técnicas existentes é a recuperação baseada por conteúdo, que possui diversos algoritmos para extração de características e usa funções de similaridade para comparar objetos diferentes. Este artigo apresenta resultados de uma Revisão Sistemática realizada sobre este tema, a fim de analisar as técnicas, extratores, funções de similaridade e banco de dados usados.

\section{Introdução}

A tecnologia vem adentrando nos últimos 20 anos em todos os setores da sociedade, mudando a forma como as pessoas trabalham e se relacionam. Os Sistemas de Informação (SI), por sua vez, estão se tornando mais complexos à medida que se fazem mais presente no cotidiano da sociedade.

Paralelamente a esse avanço tecnológico, também a área de processamento gráfico, incluindo processamento de imagens, computação gráfica, realidade virtual e áreas afins, evoluiu no decorrer dos anos, à medida que o hardware se tornou mais poderoso e barato. Com isso, a aplicação dessas tecnologias está sendo mais comum na construção de Sistemas de Informação.

Um dos focos de estudo nesse contexto são os modelos tridimensionais (3D), que vêm ganhando espaço na última década. O modelo 3D pode fornecer ao usuário uma visão mais completa do objeto sob estudo devido às possibilidades de disponibilizar, além das cores e da forma, uma dimensão adicional relacionada à profundidade [Nunes et al. 2010]. 
Com o crescimento de modelos 3D atualmente armazenados em bancos de dados, são necessários mecanismos eficientes de busca. O processo de recuperação por conteúdo de modelos 3D é bastante similar ao usado para recuperação de imagens bidimensionais (2D) e é composto por: extratores, funções de similaridade e métodos de indexação. Os extratores analisam e retiram dados, muitas vezes matemáticos, das imagens. Esses dados são agrupados em vetores e armazenados em Banco de Dados. As funções de similaridade calculam a semelhança entre o vetor de características de uma imagem modelo dada como objetivo e os vetores armazenados. Os métodos de indexação auxiliam na busca de vetores de características, melhorando o desempenho do sistema [Boohm et al. 2001]

A Revisão Sistemática (RS) é uma metodologia de pesquisa desenvolvida para reunir e avaliar estudos relevantes relacionados a um determinado tema [Kitchenham 2004]. A presente RS faz parte de um projeto em andamento que tem por objetivo a implementação de técnicas de recuperação baseada por conteúdo em modelos tridimensionais médicos. Para atingir tal objetivo e considerando o grande número de algoritmos existentes para extratores e funções de similaridade, se fez necessária a elaboração da RS apresentada neste trabalho. A partir da sumarização dos resultados da pesquisa criou-se uma síntese das abordagens apresentadas na literatura.

Em outros overviews relacionados ao assunto, como em[Yubin et al. 2007], [Qin et al. 2008] e [Gao et al. 2009] a abordagem dada era focada em extratores e não houve uma metodologia para a revisão. Na presente RS pretendemos analisar, além dos descritores, outros pontos presentes no processo de recuperação por conteúdo, e também, atualizar a literatura sobre novas técnicas existentes.

Para atingir o objetivo deste artigo, a Seção 2 introduz os conceitos sobre Revisões Sistemáticas e o protocolo usado na presente pesquisa; a Seção 3 apresenta e discute os resultados obtidos e as principais inferências realizadas e na Seção 4 são relatadas as considerações finais do trabalho.

\section{Metodologia}

A metodologia empregada para a elaboração do artigo foi o uso da técnica de Revisão Sistemática (RS). A RS é uma metodologia de pesquisa desenvolvida para reunir e avaliar estudos relevantes relacionados a um determinado tema. O processo de condução da RS obedece uma sequência bem definida de passos metodológicos, seguindo protocolos desenvolvidos com raciocínio a priori, o que a diferencia de revisões comuns. Além disso, a RS segue uma estratégia de pesquisa bem definida e documentada [Kitchenham 2004].

Ela é dividida em Planejamento, que define o problema de pesquisa, os critérios de seleção de estudos e o protocolo de revisão; Condução, na qual é feita uma análise da pesquisa bibliográfica a partir dos critérios estabelecidos no protocolo, incluindo ou excluindo estudos e extraindo informações detalhadas dos mesmos e Análise dos Resultados que documenta os resultados e conclusões obtidas da RS.

\subsection{Protocolo de Revisão Sistemática}

O protocolo de Revisão Sistemática é usado para o planejamento da revisão bibliográfica e garante que ela seja feita com o mesmo rigor de uma pesquisa. É encorajado que o protocolo seja feito em conjunto com um profissional experiente da área que pode oferecer alguns trabalhos iniciais para conhecimento do assunto, na chamada análise exploratória. 
E, também, pode avaliar se as questões de pesquisa e itens de exclusão e inclusão são pertinentes ao assunto.

O protocolo de pesquisa apresentado a seguir se baseou nos conceitos propostos por [Kitchenham 2004] e [Biolchini et al. 2005].

- Objetivos: (i) analisar, por meio de pesquisas bibliográficas, quais métodos de extração de características e funções de similaridade estão sendo usados para a recuperação de imagens tridimensionais por conteúdo; (ii)avaliar os estudos experimentais realizados recentemente envolvendo recuperação de modelos.

- Questões de Pesquisa: "Quais extratores de características e funções de similaridade são implementados para recuperação de modelos tridimensionais por conteúdo?"e 'Quais outras técnicas são empregadas para aperfeiçoar a recuperação por conteúdo de modelos 3D?".

- Fontes utilizadas: foram selecionadas bases importantes e conhecidas da área de Ciência da Computação disponíveis na web, a saber: IEEE Xplore Digital Library, ACM Digital Library e Springer.

- Critérios de Inclusão: para serem incluídos, os trabalhos deveriam: (i) conceituar extratores e/ou funções de similaridade para recuperação por conteúdo de modelos 3D ou (ii) aplicar tais técnicas em modelos 3D.

- Critérios de Exclusão: foram descartados trabalhos qu: (i) utilizam recuperação por conteúdo para imagens 2D ou (ii) não utilizam o processo de recuperação baseada por conteúdo.

- Palavras-chave: considerando que durante a análise exploratória os extratores mais comuns encontrados para esse escopo de pesquisa eram baseados em forma, adicionou-se a palavra shape às palavras-chave, além disso, foram feitas combinações do termo 3D relacionado com as palavras 3D model retrieval, shape descriptor e content-based. Para cada base de dados foi utilizada uma combinação diferente dessas palavras:

- ACM Digital Library: ("3d model retrieval" OR "content-based 3D" OR "3D shape retrieval”) AND "Abstract”: "descriptor" AND NOT "Abstract: "2D"AND NOT "semantic"

- IEEE Xplore Digital Library:("content based 3d" "3d model retrieval" “content- based 3d") NOT " $2 D$ " NOT "table" NOT "benchmark" NOT "plan*”

- Springer: :(("3d model retrieval”or "content-based 3D”) and "shape descriptor”)

\subsection{Condução da Revisão Sistemática}

Após finalizado o Protocolo, foi iniciada a fase de condução ou execução da RS. Nessa fase foram realizadas as pesquisas nas fontes listadas, utilizando-se as combinações de palavras-chave referentes a cada uma delas, a fim de encontrar trabalhos relevantes relacionados ao tema. Inicialmente, a condução foi feita no primeiro semestre de $2011 \mathrm{e}$ atualizada em Dezembro de 2011, para verificar se houve artigos relevantes para a pesquisa publicados durante o ano.

Depois, para cada uma das fontes, foram preenchidos Formulários de Condução, que registraram os trabalhos encontrados. Foram analisados os títulos, os resumos desses 
trabalhos para determinar quais trabalhos seriam incluídos e quais seriam descartados. Essa escolha é feita com base nos critérios de inclusão e exclusão citados anteriormente. A Tabela 1 mostra como os artigos foram categorizados de acordo com os critérios de Inclusão e Exclusão.

Após definição dos trabalhos incluídos, cada um deles foi lido integralmente e foram preenchidos Formulários de Extração, contendo o nome do artigo, os autores, a data de publicação, o veículo de publicação, a fonte, o resumo, descrição do método de extração/função de similaridade, qual a base de dados usada, qual a forma e métrica empregada para avaliação dos resultados, comentários adicionais e referências relevantes. Esses formulários auxiliaram a última fase da RS (Análise dos Resultados) por terem os dados extraídos dos trabalhos de forma mais detalhada.

Nas bases pesquisadas, foram encontrados 44 trabalhos, dos quais 33 foram incluídos. A Tabela 1 apresenta os artigos incluídos e quais critérios de inclusão e exclusão foram satisfeitos.

Tabela 1: Critérios de Inclusão e Exclusão atendidos

\begin{tabular}{|l|l|l|}
\hline Artigo & Itens de inclusão & Itens de exclusão \\
\hline \hline 1.[Chen et al. 2011] & $\mathrm{I} 1, \mathrm{I} 2$ & - \\
\hline 2.[Yachun et al. 2009] & $\mathrm{I} 1, \mathrm{I} 2$ & - \\
\hline 3.[Lv et al. 2009] & $\mathrm{I} 2$ & - \\
\hline 4.[Song and Golshani 2003] & $\mathrm{I} 1, \mathrm{I} 2$ & $\mathrm{E} 1$ \\
\hline 5.[Leng et al. 2007] & $\mathrm{I} 1, \mathrm{I} 2$ & - \\
\hline 6.[Khe et al. 2007] & $\mathrm{I} 1, \mathrm{I} 2$ & - \\
\hline 7.[Osada et al. 2008] & $\mathrm{I} 1, \mathrm{I} 2$ & - \\
\hline 8.[Qin et al. 2008] & $\mathrm{I} 1, \mathrm{I} 2$ & - \\
\hline 9.[Wei et al. 2008] & $\mathrm{I} 1, \mathrm{I} 2$ & - \\
\hline 10.[Ruan et al. 2008] & $\mathrm{I} 2$ & - \\
\hline 11.[Zhi-yong and Bai-lin 2010a] & $\mathrm{I} 2$ & - \\
\hline 12.[Zhi-yong and Bai-lin 2010b] & $\mathrm{I} 1, \mathrm{I} 2$ & - \\
\hline 13.[Yubin et al. 2007] & $\mathrm{I} 1, \mathrm{I} 2$ & \\
\hline 14.[Gao et al. 2009] & $\mathrm{I} 2$ & - \\
\hline 15.[Leng et al. 2007] & $\mathrm{I} 1, \mathrm{I} 2$ & - \\
\hline 16.[Li and Johan 2010] & $\mathrm{I} 1, \mathrm{I} 2$ & - \\
\hline 17.[Wang and Cui 2004] & $\mathrm{I} 1, \mathrm{I} 2$ & - \\
\hline 18.[Xiang et al. 2007] & $\mathrm{I} 1, \mathrm{I} 2$ & - \\
\hline 19.[Gong et al. 2009a] & $\mathrm{I} 1, \mathrm{I} 2$ & - \\
\hline 20.[Gong et al. 2009b] & $\mathrm{I} 1, \mathrm{I} 2$ & - \\
\hline 21.[Kazhdan et al. 2003] & $\mathrm{I} 1, \mathrm{I} 2$ & - \\
\hline 22.[Li et al. 2008a] & $\mathrm{I} 1, \mathrm{I} 2$ & - \\
\hline 23.[Li et al. 2008b] & $\mathrm{I} 1, \mathrm{I} 2$ & $\mathrm{E} 1$ \\
\hline 24.[Wu et al. 2009] & $\mathrm{I} 1, \mathrm{I} 2$ & - \\
\hline 25.[Yoon et al. 2010] & $\mathrm{I} 1, \mathrm{I} 2$ & $\mathrm{I} 1, \mathrm{I} 2$ \\
\hline 26.[Li and Johan 2011] & $\mathrm{I} 2$ & \\
\hline 27.[Wang et al. 2011] & & - \\
\hline
\end{tabular}




\begin{tabular}{|l|l|l|}
\hline 28.[Gao et al. 2011] & $\mathrm{I} 1$ & - \\
\hline 29.[Li et al. 2011] & $\mathrm{I} 1$ & - \\
\hline 30.[Fisher et al. 2011] & $\mathrm{I} 1, \mathrm{I} 2$ & - \\
\hline 31.[Axenopoulos et al. 2011] & $\mathrm{I} 1$ & - \\
\hline 32.[Saavedra et al. 2011] & $\mathrm{I} 1, \mathrm{I} 2$ & - \\
\hline 33.[Ohbuchi et al. 2008] & $\mathrm{I} 1, \mathrm{I} 2$ & $\mathrm{E} 1$ \\
\hline \hline
\end{tabular}

\section{Resultados e Discussões}

Dos trabalhos incluídos $62 \%$ implementam algum tipo de novo extrator, enquanto os outros $28 \%$ se dividem em trabalhos relacionados a overviews da área, técnicas de ajuste de pesos de extratores e algoritmos para implementar a técnica de Realimentação por Relevância (essa técnica consiste no uso do feedback do usuário para aperfeiçoar os resultados da busca). A distribuição dos artigos no decorrer dos anos é apresentada no gráfico disponibilizado na Figura 1, que mostra um maior número de publicações a partir do ano de 2008. É possível observar que em 2011 o crescimento continuou indicando que área ainda está sendo explorada, porém a análise dos artigos permitiu perceber que há tópicos de pesquisa ainda pouco explorados. A data de publicação dos trabalhos não foi um critério de inclusão e notou-se que dos artigos incluídos os primeiros eram do ano de 2003 .

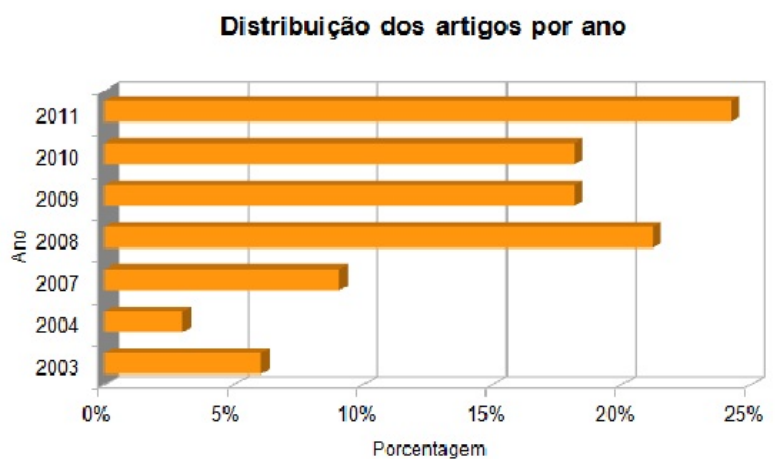

Figura 1. Distribuição de artigos por ano

Foi percebido também que em $81 \%$ dos artigos são feitos testes para avaliar o desempenho dos métodos de recuperação e, para isso, são feitas comparações com extratores que os autores julgaram mais usados e aceitos pela comunidade. Não há um consenso em relação a quais extratores podem ser usados como referência para teste. O extrator Light Field Descriptor foi o mais citado, aparecendo em 13\% dos trabalhos.

$\mathrm{Na}$ fase de pré-processamento, os modelos são normalizados de acordo com suas coordenadas canônicas [Yubin et al. 2007]. Entretanto, Kazhdan et al. (2003) propõem um novo método de normalização com coordenadas esféricas e, segundo o experimento conduzido pelos pesquisadores, o uso dessa técnica trouxe melhores resultados para o usuário. Em [Axenopoulos et al. 2011] é utilizada uma técnica de alinhamento através da estimação de posição que também se mostrou eficiente. 


\subsection{Extratores}

Por ser um tema relativamente novo, ainda não se tem uma taxonomia própria para as diferentes categorias dos extratores. Fazendo uma adaptação do modelo proposto por Qin et al. (2008), é possível dividir os extratores em globais e locais. Dos artigos estudados, $64 \%$ eram globais e 36\% locais. Como ressalta Gao et al (2009), percebe-se que novos esforços estão sendo dirigidos para implementação de técnicas para recuperação de modelos baseados em dados parciais dos mesmos. Além disso, acredita-se que os extratores globais são mais robustos e fáceis de implementar [Qin et al. 2008].

Dentro dessas categorias, os extratores ainda podem ser divididos em Shape-based e View-based. Como os nomes sugerem, os primeiros extraem características referentes à forma, volume e contorno do modelo, enquanto a segunda categoria se caracteriza por extratores que analisam textura, cores e fazem projeções dos modelos [Qin et al. 2008].

Em relação aos artigos analisados, os autores implementaram extratores locais, partindo do princípio que os modelos possuem particularidades que são ignoradas em uma análise global. Em [Osada et al. 2008] foi implementado um extrator que calcula o volume em diferentes partes dos modelos. Já em [Gong et al. 2009b] e [Wu et al. 2009] é apresentado uma análise do grau de interconectividade de determinado voxel e seus vizinhos.

Os artigos de [Li and Johan 2010], [Li et al. 2011] e [Saavedra et al. 2011] se destacam por serem baseados em projeções e implementarem funções que analisam contornos. Já em [Leng et al. 2007] é aplicado o descritor de Tchebichev nas projeções extraídas.

Os trabalhos de [Gong et al. 2009a], [Yachun et al. 2009], [Song and Golshani 2003], [Khe et al. 2007] e [Wang and Cui 2004] apresentam o desenvolvimento de extratores globais. Em [Khe et al. 2007] e [Yachun et al. 2009] são desenvolvidos extratores baseados em forma que se utilizam de histogramas de distância. Já em [Song and Golshani 2003], [Gao et al. 2011] e [Wang and Cui 2004] são utilizados métodos para rotular os modelos a fim de auxiliar na busca e no mapeamento geométrico. Por fim, o trabalho de [Gong et al. 2009a] se utiliza do descritor de Fourier e Máquinas de Aprendizado Supervisionado para recuperar faces humanas similares.

O trabalho de [Wei et al. 2008] foi o único encontrado nessa Revisão Sistemática que se utilizou apenas das cores dos modelos para realizar a busca. A Figura 2 ilustra como se deu a distribuição por tipo de extrator implementado no conjunto de artigos analisados.

\subsection{Funções de Similaridade e Métricas de Avaliação}

Em relação às funções de Similaridade, a Distância Euclidiana foi a mais usada, apesar de ser afirmado que essa métrica, assim como a Distância Manhattan, não são boas para analisar vetores de características, uma vez que ela é sensível a valores discrepantes de extratores [Yubin et al. 2007]. A Figura 3 ilustra a distribuição do uso de diferentes funções de distância nos artigos encontrados.

Estudos na área de recuperação de imagens bidimensionais indicam que em testes realizados com imagens mamográficas com diferentes funções de similaridade, mostraram que as métricas como Euclidiana, Manhattan e Chebychev são mais sensíveis 


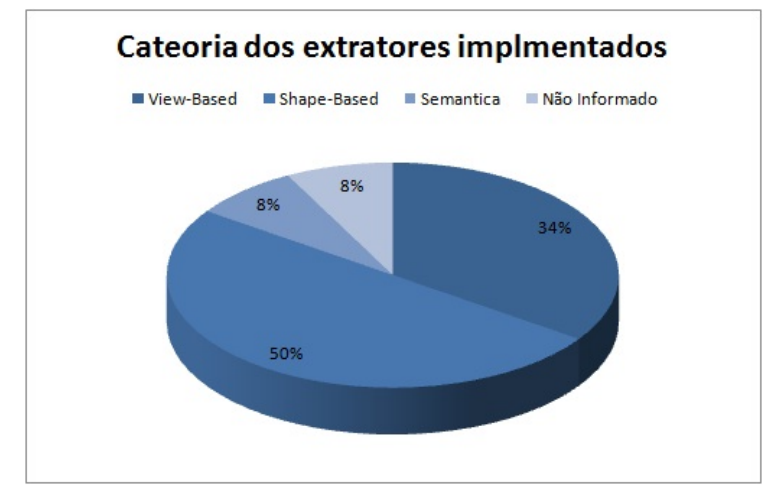

Figura 2. Tipos dos extratores implementados

à variação significativa em apenas uma característica. Já as técnicas Canberra, Trigonométrica Modifcada, $X^{2}$ e Divergência de Jeffrey mostraram-se menos sensíveis e mais tolerantes a ruídos nos vetores de características [Goncalves et al. 2011].

Alguns autores optaram por agrupar os vetores de características em clusters e dessa forma, usaram técnicas como k-means, redes de Hopfield e Máquinas de Aprendizado Supervisionado para realizar o agrupamento e o cálculo de dispersão dos grupos. Já a Kullback-Leibler é uma medida estatística que calcula a divergência de determinada distribuição probabilística.

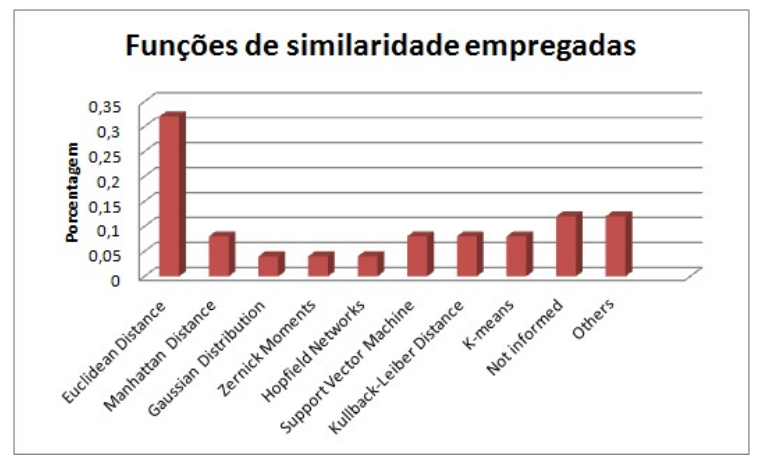

Figura 3. Funções de Similaridade usadas

Outro aspecto para o qual foi percebida bastante uniformidade nos resultados encontrados foi em relação à metrica de avaliação do sistema de recuperação propriamente dito. A mais usada foi a Precisão versus Revocação, uma medida já bastante utilizada no contexto de recuperação por conteúdo em imagens bidimensionais, e que talvez por esse motivo, se estendeu para os modelos 3D. Ela foi usada em 37\% dos trabalhos, como em [Li et al. 2008a], [Wang et al. 2011], [Li et al. 2008b] e [Xiang et al. 2007], sendo que no restante houve diversas métricas implementadas, porém nenhuma tendo uma utilização tão alta. A Figura 4 mostra esse cenário.

Enquanto a métrica de Precisão versus Revocação envolve somente esses dois atributos, a Média de Precisão envolve também o ranking de relevância das imagens recuperadas. A métrica Normalized Recall, como o nome sugere, normaliza o atributo revocação em relação aos itens relevantes. Nearest Neighbor, First-Tier e Second-Tier são métricas que analisam a quantidade de modelos que foram dados como consulta com os 
$K$ primeiros modelos retornados.

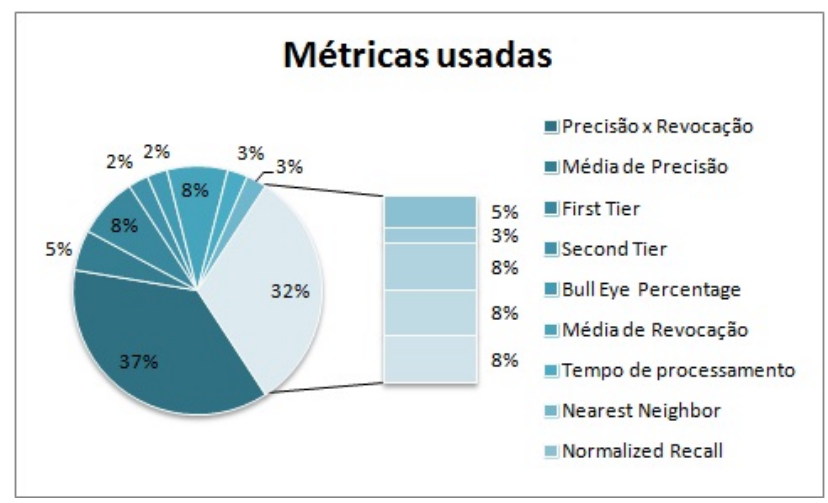

Figura 4. Técnicas de avaliação usadas

\subsection{Base de Dados}

Essa informação era uma das mais importantes para o contexto de todo o projeto, visto que esperava-se que a partir de informações encontradas nos artigos fosse possível mapear alguma base de imagens médicas que fosse pública e que fornecesse índices para comparar extratores. No entanto, foi notado que em $42 \%$ dos artigos foi utilizada a base de dados Princeton Benchmark, uma base de dados genérica, como em [Gong et al. 2009b], [Yoon et al. 2010], [Li and Johan 2011], [Ohbuchi et al. 2008], entre outros. Um dado alarmante foi que $41 \%$ dos trabalhos não indicaram qual a sua fonte de dados, o que pode ser considerado uma falha aos resultados encontrados dessas pesquisas.

O fato da principal base encontrada ser genérica mostra que as pesquisas nesse campo de conhecimento ainda estão em sua fase inicial, uma vez que os os pesquisadores estão implementando a recuperação por conteúdo em modelos simples. Espera-se que com a evolução das técnicas, os extratores se tornem mais específicos e elaborados, fornecendo, de fato, utilidade para a aplicação real de recuperação de modelos tridimensionais no cotidiano dos Sistemas de Informação.

\subsection{Outras técnicas}

Outras técnicas encontradas e que podem ser úteis para a pesquisa na qual esta RS está inserida são referentes à Realimentação por Relevância, na qual, por meio do feedback do usuário, é possível ajustar valores de pesos entre os modelos, refinando a consulta.

Nos trabalhos de [Zhi-yong and Bai-lin 2010a] e [Zhi-yong and Bai-lin 2010b] são construídas funções de regressão com base no retorno dos usuários e ajustando-se os pesos dos modelos com Regressão Logística e Hidden Markov Models, respectivamente.

Há também algoritmos que combinam diferentes extratores a fim de obter um melhor resultado de busca, avaliado por meio de métricas como "Precisão versus Revocação"( [Chen et al. 2011], [Lv et al. 2009], [Leng et al. 2007]).

O trabalho de [Chen et al. 2011] se destaca por utilizar Lógica Fuzzy para criar regras e adaptar os pesos de acordo com o feedback do usuário. Na pesquisa de [Fisher et al. 2011], os pesquisadores transformam uma cena tridimensional em grafo e a busca por conteúdo se dá em função dos itens dispostos em determinada cena. Por 
exemplo, para encontrar uma mesa para escritório foi dada como query diversos itens relacionados à mesa, como computador, caderno e caneta.

Por fim, é importante destacar que o desempenho da busca pode ser influenciado pela sua infraestrutura, como mostra o trabalho de [Ruan et al. 2008], no qual os autores concluem que o número de processadores influencia no tempo de processamento de extração de características no retorno dos resultados da busca. No experimento foram usados 8, 10, 12 e 14 processadores para efetuar a busca de 500, 1000 e 1500 modelos. Em todos os casos o tempo diminuiu conforme o número de processadores aumentou.

\section{Conclusões}

Neste artigo foram apresentados a condução e os resultados de uma Revisão Sistemática, realizada com a finalidade de analisar diversas técnicas envolvidas no processo de recuperação de modelos tridimensionais.

Em linhas gerais, pode-se dizer que a Revisão Sistemática foi eficiente por sistematizar o método de revisão bibliográfica e permitir melhorar o escopo da pesquisa.

No contexto de Sistemas de Informação, foi visto que as técnicas implementadas foram em sua totalidade usadas somente em pequenos experimentos em um cenário totalmente controlado. Não foram identificados trabalhos que usassem os métodos desenvolvidos para auxiliar na solução de algum problema específico e real. Isso acaba se tornando um novo paradigma para a área criando novas oportunidades, uma vez que a indústria demanda cada vez mais por soluções aplicadas aos seus problemas, investindo em setores como Pesquisa e Desenvolvimento.

Com os resultados encontrados, é visto que o tema de recuperação por conteúdo em modelos tridimensionais é bastante novo, com artigos sendo publicados a partir de 2003, mas a maioria estando concentrada a partir de 2008. Foi visto que por ser um assunto novo não existem extratores que sirvam como referência e nem uma taxonomia própria e há, ainda, um espaço grande na busca de modelos 3D tendo somente parte deles como argumento de busca.

Em paralelo, a sociedade tem gerado e disponibilizado cada vez mais informações em formato multimídia. Imagens, sons e filmes têm sido incluídos em diversos tipos de SI com o objetivo de identificar pessoas, objetos e locais. Da mesma forma, SI com interação e ambientes tridimensionais vêm sendo considerados em diversos ramos da indústria. Com esse crescente aumento do volume de informação armazenada, a pesquisa em recuperação de modelos tridimensionais armazenados em grandes bases de dados pode contribuir para que o acesso à informação seja facilitado e, no futuro, a interação com SIs seja mais natural. Espera-se, então, que os trabalhos relacionados à área avancem para modelos cada vez mais complexos e específicos auxiliando diversos setores da sociedade.

\section{Agradecimentos}

Os autores agradecem ao Conselho Nacional de Desenvolvimento Científico e Tecnológico (CNPq) - Processo 559931/2010-7, à Fundação de Amparo à Pesquisa do Estado de São Paulo (Fapesp) - Processo 2010/15691-0 e ao Instituto Nacional de Ciência e Tecnologia - Medicina Assistida por Computação Científica (INCT-MACC), pelo apoio financeiro. 


\section{Referências}

Axenopoulos, A., Litos, G., and Daras, P. (2011). 3d model retrieval using accurate pose estimation and view-based similarity. In Proceedings of the 1st ACM International Conference on Multimedia Retrieval, ICMR '11, pages 41:1-41:8, New York, NY, USA. ACM.

Biolchini, J., Mian, P. G., Natali, A. C. C., and Travassos, G. H. (2005). Systematic review in software engineering. In Systems Engineering and Computer Science Department.

Boohm, C., Berchtold, S., and Keim, D. A. (2001). Searching in high-dimensional spaces: index structures for improving the performance of multimedia databases. ACM Computing Surveys, $33: 322-373$.

Chen, Z. Q., Zou, K. S., Ip, W. H., and Chan, C. Y. (2011). 3d model retrieval based on fuzzy weighted shape distributions. In Advanced Materials Research, volume 201-203, pages 16781681. Trans Tech Publications.

Fisher, M., Savva, M., and Hanrahan, P. (2011). Characterizing structural relationships in scenes using graph kernels. ACM Trans. Graph., 30:34:1-34:12.

Gao, B., Zheng, H., and Zhang, S. (2009). An overview of semantics processing in content-based $3 \mathrm{~d}$ model retrieval. In Proceedings of 11 th International Conference on Artificial Intelligence and Computational Intelligence. AICI, number 11, pages 54-59, Shanghai, China. IEEE Computer Society.

Gao, Y., Wang, M., Zha, Z.-J., Tian, Q., Dai, Q., and Zhang, N. (2011). Less is more: Efficient 3-d object retrieval with query view selection. Multimedia, IEEE Transactions on, 13(5):1007 -1018 .

Goncalves, V. M., Nunes, F. S., Delamaro, M. E., and Oliveira, R. A. P. (2011). Avaliacao de funcoes de similaridade em um framework de teste para programas com saidas graficas. In Proceedings of XXXVII Conferencia Latinoamericana de Informatica (XXXVII CLEI), volume 1, pages $1-15$.

Gong, B., Wang, Y., Liu, J., and Tang, X. (2009a). Automatic facial expression recognition on a single $3 \mathrm{~d}$ face by exploring shape deformation. In Proceeding in 17th ACM international conference on Multimedia, number 17, pages 569-572, Beijing, China. ACM - Association for Computing Machinery.

Gong, B., Xu, C., Liu, J., and Tang, X. (2009b). Boosting 3d object retrieval by object flexibility. In Proceeding in 17th ACM international conference on Multimedia, number 17, pages 525528, Beijing, China. ACM - Association for Computing Machinery.

Kazhdan, M., Funkhouser, T., and Rusinkiewicz, S. (2003). Rotation invariant spherical harmonic representation of $3 \mathrm{~d}$ shape descriptors. In Proceedings of the 1th Eurographics/ACM SIGGRAPH symposium on Geometry processing, number 1, pages 156-164, Aachen, Germany. Eurographics Association.

Khe, L., Feng, Z., and Ning, H. (2007). An effective approach to content-based 3d model retrieval and classification. In Proceedings of the 1th Internacional Conference on Computational Intelligence and Security (CIS), number 1, pages 361-365, China. IEEE Computer Society.

Kitchenham, B. (2004). Procedures for performing systematic review. Technical Report TR/SE0401, Keele University. 
Leng, B., Li, L., and Qin, Z. (2007). Made: A composite visual-based 3d shape descriptor. In Proceedings of 3th Internacional Conference on Computer Vision/Computer Graphics Collaboration Techniques, volume 4418, pages 93-104, Rocquencourt, France. Springer Berlin / Heidelberg.

Li, B. and Johan, H. (2010). View context: A 3d model feature for retrieval. In Proceedings of 11 th Conference on Advances in Multimedia Information Processing, volume 5916, pages 185-195. Springer Berlin / Heidelberg.

Li, B. and Johan, H. (2011). 3d model retrieval using hybrid features and class information. Multimedia Tools and Applications, pages 1-26. 10.1007/s11042-011-0873-3.

Li, L., Zhang, S., Zhang, K., and He, Q. (2011). View-based 3d cad model retrieval using local visual features. In Image and Signal Processing (CISP), 2011 4th International Congress on, volume 3, pages $1373-1376$.

Li, X., Godil, A., and Wagan, A. (2008a). 3d part identification based on local shape descriptors. In Proceedings of 8th Workshop on Performance Metrics for Intelligent Systems, number 8, pages 162-166, Washington, United States. ACM - Association for Computing Machinery.

Li, Z., Mitani, J., Fukui, Y., and Nishihara, S. (2008b). Multiresolution wavelet analysis of shape orientation for $3 \mathrm{~d}$ shape retrieval. In Proceeding of the 1th ACM international conference on Multimedia information retrieval, number 1, pages 403-410, Vancouver, Canada.

Lv, T., Liu, G., bin Huang, S., and xuan Wang, Z. (2009). Selective feature combination and automatic shape categorization of $3 \mathrm{~d}$ models. In Proceedings of 6th International Conference on Fuzzy Systems and Knowledge Discovery. FSKD, number 6, pages 447-451, Tianjin, China. IEEE Computer Society.

Nunes, F. L. S., Melo, C. R. M., Correa, C. G., Tori, R., Barbosa, J., Pichi, F., and Nakamura, R. (2010). importancia da avaliacao na engenharia de requisitos em sistemas de realidade virtual e aumentada: um estudo de caso. In Symposium on Virtual and Augmented Reality, number 7, Natal, Rio Grande do Norte.

Ohbuchi, R., Osada, K., Furuya, T., and Banno, T. (2008). Salient local visual features for shapebased 3d model retrieval. In Proceedings of 8th IEEE International Conference on Shape Modeling and Applications. SMI, number 8, pages 93-102.

Osada, K., Furuya, T., and Ohbuchi, R. (2008). Local volumetric features for 3d model retrieval. In Proceeding of 8th IEEE International Conference on Shape Modeling and Applications. SMI, number 8, pages 245-246, New York, USA. IEEE Computer Society.

Qin, Z., Jia, J., and Qin, J. (2008). Content based 3d model retrieval: A survey. In Proceedings of 6th International Workshop on Content-Based Multimedia Indexing. CBMI., number 6, pages 249-256, London, England. IEEE Computer Society.

Ruan, J., Lin, J., Yang, Y., and Wei, W. (2008). A high performance computing platform for 3d model retrieval. In Proceedings of 1th International Conference on Computer Science and Software Engineering. (CSSE), number 1, pages 446-449, Wuhan, China. IEEE Computer Society.

Saavedra, J. M., Bustos, B., Scherer, M., and Schreck, T. (2011). Stela: sketch-based 3d model retrieval using a structure-based local approach. In Proceedings of the 1st ACM International Conference on Multimedia Retrieval, ICMR '11, pages 26:1-26:8, New York, NY, USA. ACM. 
Song, J.-J. and Golshani, F. (2003). Shape-based 3d model retrieval. In Proceedings of 15th IEEE International Conference on Tools with Artificial Intelligence, number 15, pages 636640, California, Estados Unidos. IEEE Computer Society.

Wang, D. and Cui, C. (2004). 3d model similarity measurement with geometric feature map based on phase-encoded range image. In Proceedings of 5th Conference on Advances in Multimedia Information Processing, volume 3333, pages 103-110. Springer Berlin / Heidelberg.

Wang, X., Wang, S., and Pang, H. (2011). Distance metric learning based on semantic correlation strength for 3d model retrieval. In Multimedia and Signal Processing (CMSP), 2011 International Conference on, volume 1, pages 334-338.

Wei, W., Yang, Y., Lin, J., and Ruan, J. (2008). Color-based 3d model classification using hopfield neural network. In Proceedings of 1th International Conference on Computer Science and Software Engineering. CSSE, number 1, pages 883-886, Wuhan, China. IEEE Computer Society.

Wu, H.-Y., Luo, T., Wang, L., Wang, X.-L., and Zha, H. (2009). 3d shape retrieval by using manifold harmonics analysis with an augmentedly local feature representation. In Proceeding of 8th International Conference on Virtual Reality Continuum and its Applications in Industry, number 8, pages 311-314, Tokio, Japan. ACM - Association for Computing Machinery.

Xiang, P., Hua, C., Gang, F., and Chuan, Z. (2007). Pose insensitive 3d retrieval by poisson shape histogram - in computational science. In Proceedings of 7 th International Conference of Computational Science, volume 4488, pages 25-32, Beijing, China. Springer Berlin / Heidelberg.

Yachun, F., Mingquan, Z., and Guohua, G. (2009). Semantic-oriented 3d model retrieval using visual vocabulary labelling. In Proceedings of 1th International Joint Conference on Artificial Intelligence. JCAI, number 1, pages 642-647, Hainan, Island. IEEE Computer Society.

Yoon, S. M., Scherera, M., Schreck, T., and Kuijper, A. (2010). Sketch-based 3d model retrieval using diffusion tensor fields of suggestive contours. In Proceedings of the 18th international conference on Multimedia, number 18, pages 193-200, Firenze,Italy. ACM - Association for Computing Machinery.

Yubin, Y., Hui, L., and Yao, Z. (2007). Content-based 3-d model retrieval: A survey. In Proceedings of 7th IEEE Transactions on Systems, Man, and Cybernetics., volume 36, pages 10811098. IEEE Computer Society.

Zhi-yong, Z. and Bai-lin, Y. (2010a). A relevance feedback based on bayesian logistic regression for $3 \mathrm{~d}$ model retrieval. In Proceeding of 2th International Conference on Computer Application and System Modeling (ICCASM), number 2, pages 77-80, Taiwan. IEEE Computer Society.

Zhi-yong, Z. and Bai-lin, Y. (2010b). A relevance feedback scheme based on hidden markov model regression for $3 \mathrm{~d}$ model retrieval. In Proceedings of 3th International Workshop on Advanced Computational Intelligence. (IWACI), number 3, pages 657-660, Jiagsu, China. IEEE Computer Society. 